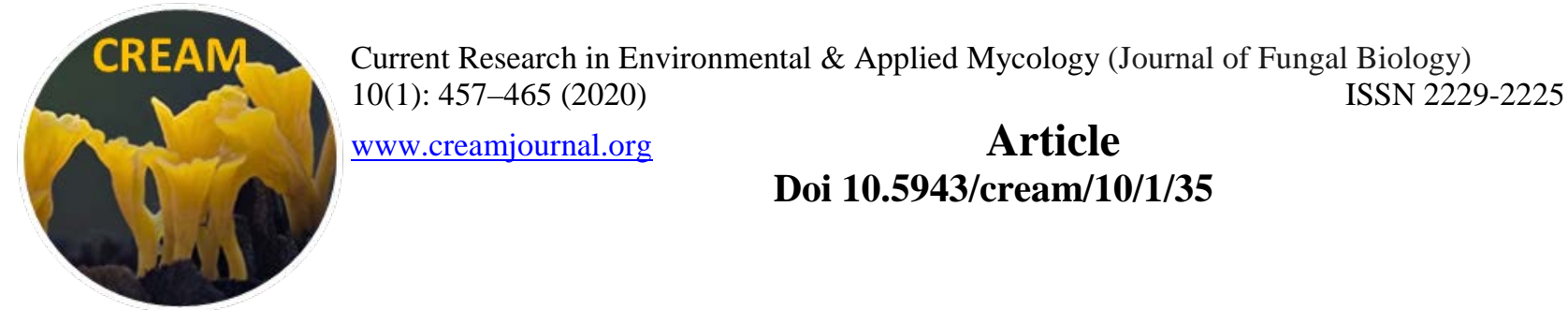

\title{
Growing of Polyporus umbellatus
}

\section{Pasailiuk MV}

Hutsulshchyna National Nature Park, 84 Druzhba St., Kosiv, Ivano-Frankivsk Obl. 78600, Ukraine

Pasailiuk MV 2020 - Growing of Polyporus umbellatus. Current Research in Environmental \& Applied Mycology (Journal of Fungal Biology) 10(1), 457-465, Doi 10.5943/cream/10/1/35

\begin{abstract}
Polyporus umbellatus is a valuable edible and medicinal fungus. Growth of two $P$. umbellatus strains 2510 and 2511 was studied on various plant substrates and their compositions. Fruit bodies of $P$. umbellatus were produced after successful mycelial colonization of the substrates with added freshly fallen oak leaves, wheat and sunflower seed shells. Using this growing method, the small fruit bodies $(2.5 \times 3.5 \mathrm{~cm}, 60-70 \mathrm{~g})$ of $P$. umbellatus, strain 2511 , developed on day 78, including 25 days of cultivation on MEA, 35 days of cultivation on substrate containing $50 \mathrm{~g}$ of wheat grain and sunflower husk with ratio 80:20, and 18 days cultivation on substrate containing $600 \mathrm{~g}$ freshly fallen oak leaves.
\end{abstract}

Key words - fruiting body - mycelial growth - plant substrate

\section{Introduction}

Polyporus umbellatus (Pers.) Fr. is a rare fungus and has been introduced to the Red Data Book of Ukraine (Didukh 2009). In the Red Lists of Estonia its conservation status is critically endangered, endangered in Croatia, vulnerable in Czech Republic, Hungary, Latvia, Norway, near threatened in Denmark, Finland, Great Britain, Sweden, and the Netherlands, according to the IUCN Red List Categories. This fungus is widely distributed in East Asia, Europe and North America (Bohlin et al. 2006, Kunca 2011, Bisko et al. 2018).

This mushroom is commonly known as "Zhu Ling” in China and widely used in traditional Chinese medicine for centuries. Its young fruiting bodies are regarded as edible. The fruiting body of P. umbellatus is composed of numerous caps (Bandara et al. 2015). The multiple circular pilei arising from a common stem make its very distinct species (Ryvarden \& Melo 2014). The fruit bodies are whitish, becoming brown with age, their characteristics pores are circular to angular (Ryvarden \& Gilbertson 1994, Stamets 2002). This fungus usually grows at the base of trunks, roots and stumps of oak, birch in the deciduous and mixed forests from June to October (Kotlaba 1984), forming underground sclerotia (Zhao \& Zhang 1992, Guo et al. 2019). The sclerotia have been used to treat edema and promote diuretic processes (Ying et al. 1987). They contain the polysaccharides that promote the antitumor and immunomodulating activities (Yang et al. 2004, Zeng et al. 2011). This fungus has a diuretic effect on pathogenic dampness and it is being used in traditional medicine combinations to treat oliguria, diarrhea, acute nephritis, thirst, urination disturbance, sunstroke, jaundice, cirrhosis, and ascites (Ying et al. 1987, Wu 2005, Liu \& Liu 2009, Zhao et al. 2009). The sclerotia of $P$. umbellatus were successfully cultured in China with Armillaria mellea (Vahl) P. Kumm, both on the artificial media and under natural conditions using burying and root inoculation methods (Choi et al. 2003, Lee et al. 2013). 
While the sales commercial products of sclerotia and fruit bodies of $P$. umbellatusre trend to increase, the resources of $P$. umbellatus in the wild will soon exhaust (Guo et al. 2002). Therefore it is necessary to cultivate the $P$. umbellatus under artificial or semi artificial methods (Huang \& Liu 2007, Xing et al. 2013). In this study, we aim to test the new cultivation methods for $P$. umbellatus productions by using the composition of various plant substrates without $A$. melea.

\section{Materials \& Methods}

\section{Mycelial culture}

The pure cultures of $P$. umbellatus strains 2510 and 2511 were obtained from the Mushroom Culture Collection of the M.G. Kholodny Institute of Botany (IBK) of the National Academy of Sciences of Ukraine (Bisko et al. 2016, 2018, World Data Center for Microorganism 2019).

\section{Culture growth on nutrient agar medium}

Polyporus umbellatus culture was isolated onto malt extract agar (MEA), $\mathrm{pH} \mathrm{6}$, and incubated at $26^{\circ} \mathrm{C}$ for 25 days (Bisko et al. 2016). The radial growth rate of the mycelium ( $\mathrm{mm} /$ day) was calculated according to Lomberg \& Solomko (2012).

\section{Spawn preparation on plant substrates}

Polyporus umbellatus spawn was prepared in two consecutive stages, including the different substrates and their combinations (Table 1).

Table 1 Substrate compositions.

\begin{tabular}{llllll}
\hline \multirow{2}{*}{$\begin{array}{l}\text { Components of the substrates } \\
\text { (\%) }\end{array}$} & WgSs & WgBsWsPs & wheat & leaf & WsOl \\
\cline { 2 - 6 } & 80 & 50 & 100 & - & - \\
Wheat grain (Wg) & 20 & - & - & - & - \\
Sunflower seed shells (Ss) & - & 25 & - & - & - \\
Beech shavings (Bs) & - & 12.5 & - & - & 50 \\
Wheat straw (Ws) & - & 12.5 & - & - & - \\
Peanut shells (Ps) & - & - & - & 100 & 50 \\
Oak leaves (Ol) & & &
\end{tabular}

Notes - WgSs: wheat grain/sunflower seed shells, WgBsWsPs: wheat grain/beech shavings/wheat straw/peanut shells, wheat: wheat grain, leaf: oak leaves, WsOl: wheat straw/oak leaves

Substrates components were cooked as described by Pasailiuk et al. (2019). Wheat grain (10 $\mathrm{kg}$ ) was cooked for 25-30 min in 10 liters of water. After drying, one kilograms of wheat grain was mixed with $12 \mathrm{~g}$ of gypsum $\left(\mathrm{CaSO}_{4} \cdot 2 \mathrm{H}_{2} \mathrm{O}\right)$ and $3 \mathrm{~g}$ of chalk $\left(\mathrm{CaCO}_{3}\right)$. Sunflower seed shells and peanut shells were pre-dried. Beech shavings (Fagus sylvaticus) was obtained during the shredding of healthy beech wood and the size of the wooden particles was $10 \times 10-40 \times 1 \mathrm{~mm}$. Wheat straw was dried and chopped to $2.5-5 \mathrm{~cm}$ particles size. Forest litters were collected under the oak (Quercus robur) and dried. All substrates were moisturized to $60 \%$ humidity, and filled to 0.5 liters glass jars and flasks (WgSs, WgBsWsPs, wheat substrates) or put into 4 liters polyethylene bags (WsOl and leaf substrates). Fifty grams of each WgSs, WgBsWsPs and wheat substrate were put into the jars and flasks, autoclaved at $121^{\circ} \mathrm{C}$ for $90 \mathrm{~min}$. Each jar or flask was inoculated with 1/4 of the mycelial colony on a $90 \mathrm{~mm}$ petri dish and incubated at $26^{\circ} \mathrm{C}$ for 35 days.

Six hundred grams of each leaf substrate and $\mathrm{WsOl} \mathrm{(pH} 4.6$ and 5.5) were autoclaved twice at $121^{\circ} \mathrm{C}$ for 1 hour. After cooling down, $1 / 3$ part of the 35 days old culture of $P$. umbellatus were transferred to each substrate and incubated at $22^{\circ} \mathrm{C}$, under the light maximum 200 lux/darkness for 12:12 hours, $75 \%$ humidity content, and ventilated at $1-3$ room volumes air exchange per hour. The mycelium colonization rate of each substrate was monitored daily and recorded by measuring of the height of the mycelium which covered the substrate from four mutually perpendicular sides. The 
average values were calculated by adding four values of heights and dividing by four and the \% of colonization the substrate by mycelium was determined as:

$$
\text { Percentage of colonization }(\%)=\left(h_{r}+h_{l}+h_{a}+h_{f}\right) / h_{s} \times 100 \%
$$

Where:

$h_{r}, h_{l}, h_{a}, h_{f}-$ height of mycelium covering the right, left, back and front side of jar/flask/bag, correspondently, $\mathrm{h}_{\mathrm{s}}$ - height of substrate in the jar/flask/bag.

Macro-morphological features of the mycelium were described according to the standard methods proposed by Stalpers (1978). The morphological characteristics at day 15 on MEA media, day 19 on the wheat substrate, day 28 on the WgSs, WgBsWsPs, and wheat substrates, and day 23 on leaf and WsOl substrates were recorded.

\section{Primordia and fruiting bodies of Polyporus umbellatus on plant substrates}

The primordia and young fruit bodies of $P$. umbellatus 2511 on the $\mathrm{WsOl}$ and leaf substrates were recorded. Their morphology, color, size and location in the substrates were described.

\section{Statistical analysis}

Data analysis was carried out using Statistica 8.0 software (StatSoft Inc., USA). Significant differences between values are indicated at $p \leq 0.05$.

\section{Results}

\section{Mycelial growth rate and morphology of Polyporus umbellatus}

The growth rate of $P$. umbellatus was $2.97-3.01 \mathrm{~mm} /$ day on nutrient media, thus this fungus belongs to slow-growing cultures (2-4 mm/day). The mycelial morphology of $P$. umbellatus colonies of the strains 2510 and 2511 on MEA was dense, opaque, white wooly, with concentric circles formed with cottony aerial hyphae (Fig. 1a, b). The colony margin is raised above the substrate, and became yellow after incubation for 35 days.

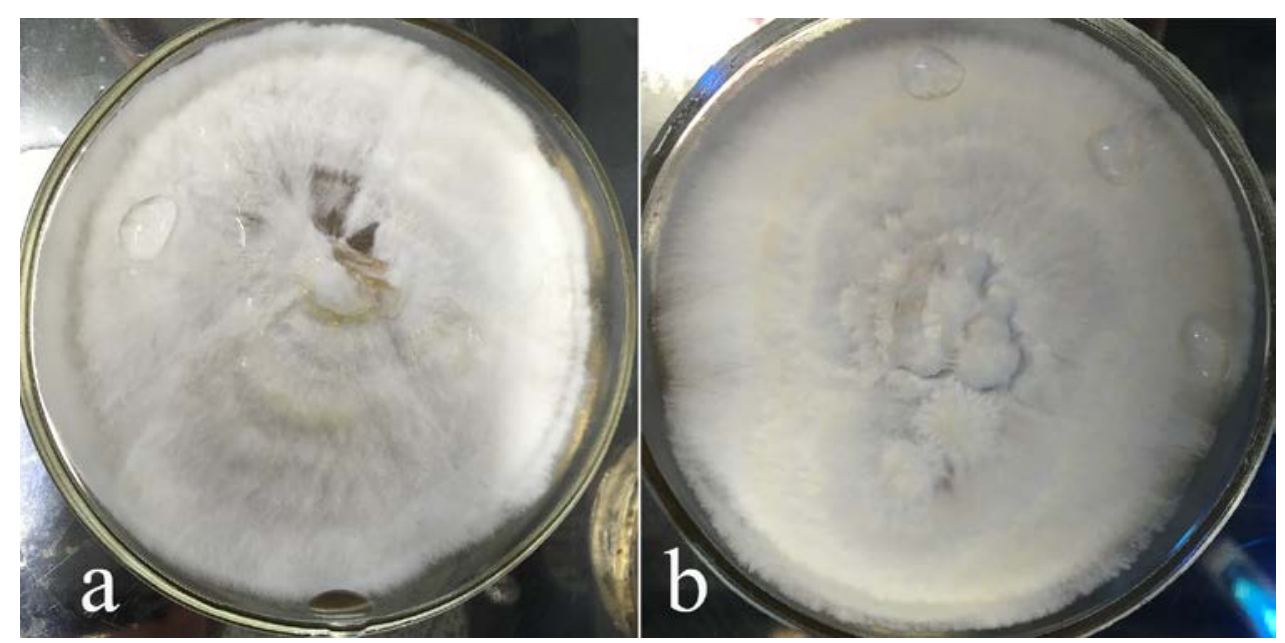

Fig. 1 - Polyporus umbellatus mycelial colonies incubated at $26^{\circ} \mathrm{C}$ for 15 days. a strain 2510 . b strain 2511.

\section{Morphology and growth rate of Polyporus umbellatus on WgSs, WgBsWsPs and wheat substrates}

After incubation for 21 days, strain 2510 was growing better on WgSs substrate (Fig. 2), while strain 2511 was able growth well on wheat media (Fig. 3). 
The WgSs and WgBsWsPs substrates were equally suitable for the mycelial growth of $P$. umbellatus. Also, these two substrates were significantly less susceptible to contamination compared to wheat (5\% vs $25 \%$ ). No significant differences were observed in the mycelial morphology of strains grown on different substrates. The mycelial colonies had distinctive cotton white and fluffy surface (Figs 4, 5).

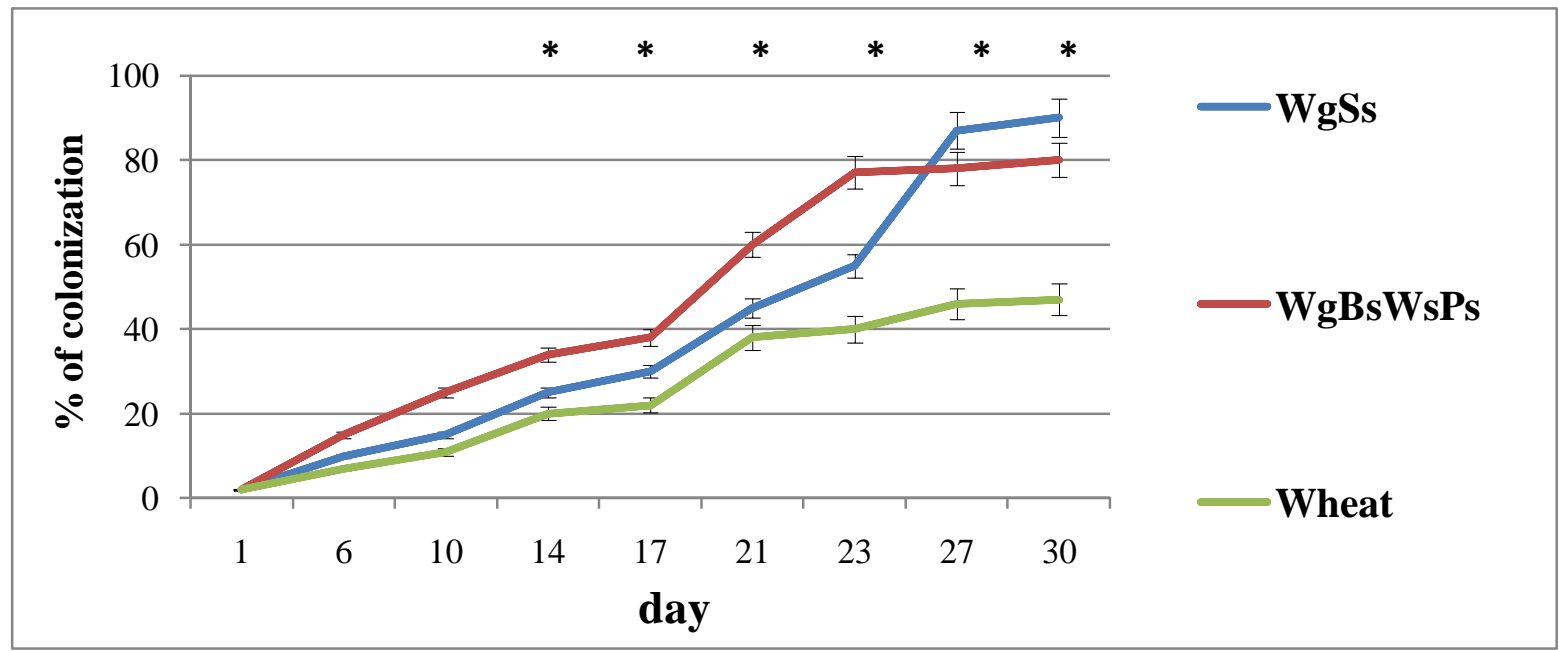

Fig. 2 - Colonization rate of Polyporus umbellatus strain 2510 on various substrates.

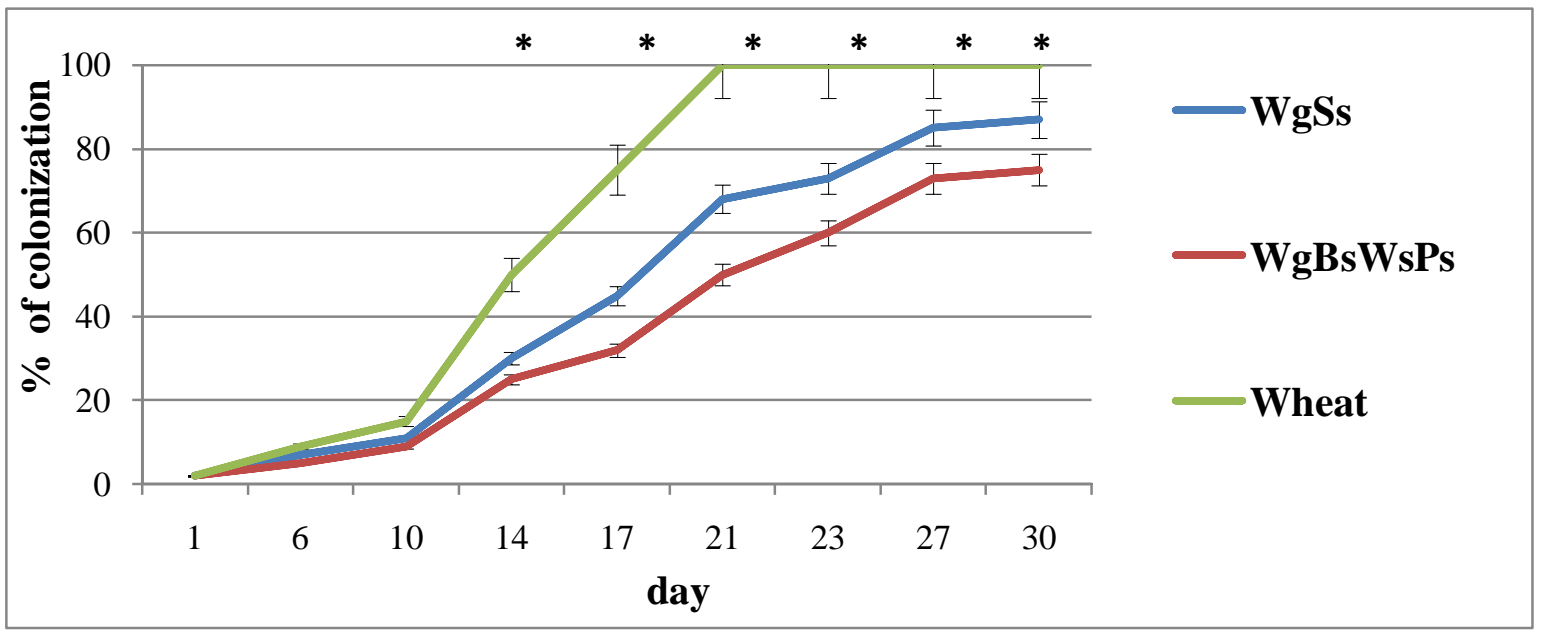

Fig. 3 - Colonization rate of Polyporus umbellatus strain 2511 on various substrates.

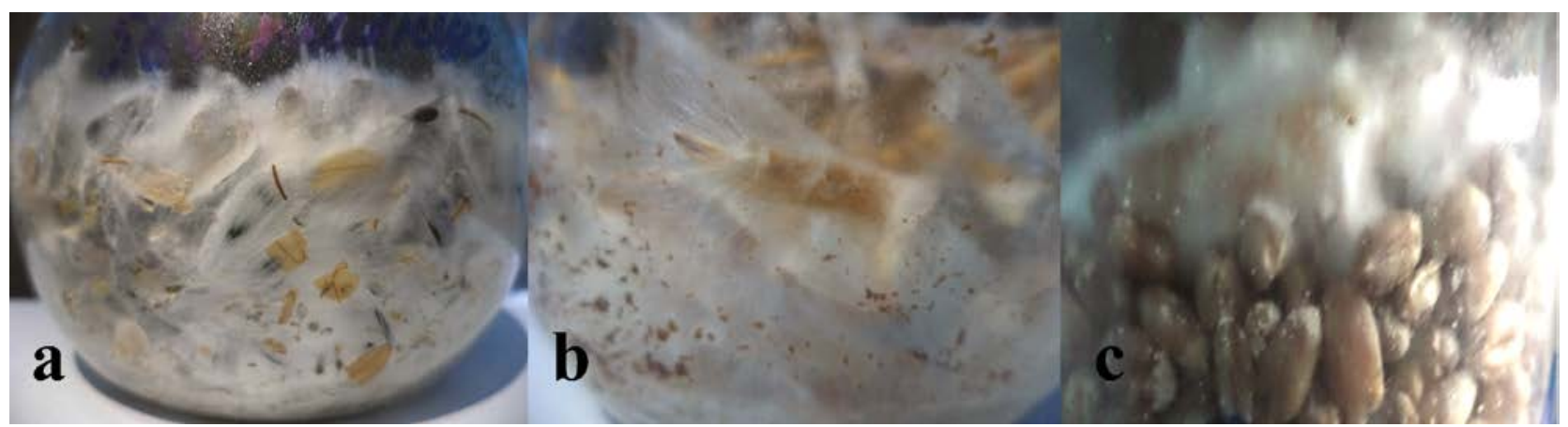

Fig. 4 - Polyporus umbellatus strain 2510 incubated on different substrates for 28 days. a WgSs substrate. b WgBsWsPs substrate. c Wheat substrate. 


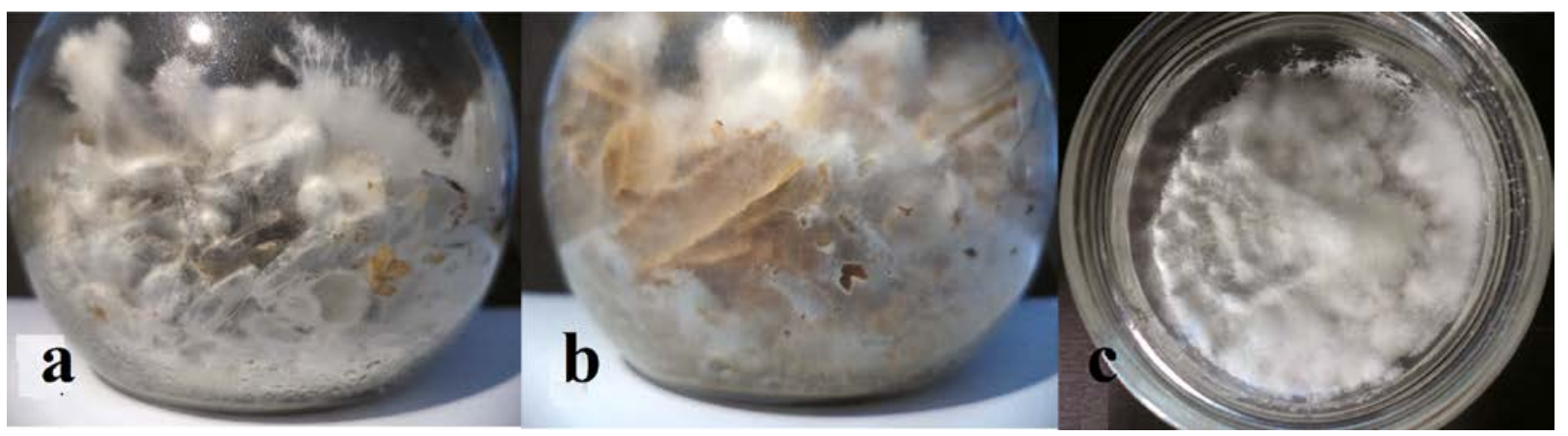

Fig. 5 - Polyporus umbellatus strain 2511 incubated on different substrates. a WgSs substrate after incubation for 28 days. b WgBsWsPs substrate after incubation for 28 days. c Wheat substrate incubated for 19 days.

Substrate, colonized with mycelium, was not firmly attached to the jar wall (Fig. 6a, b, c), as it was in the flasks. Therefore its removing from the flasks (Fig. 6f) was hard, but its removing from the jars (Fig. 6d, e) was easy, not damaging the mycelium structure and did not take long time, thus minimizing the mycelium exposure to the exogenous environmental factors.

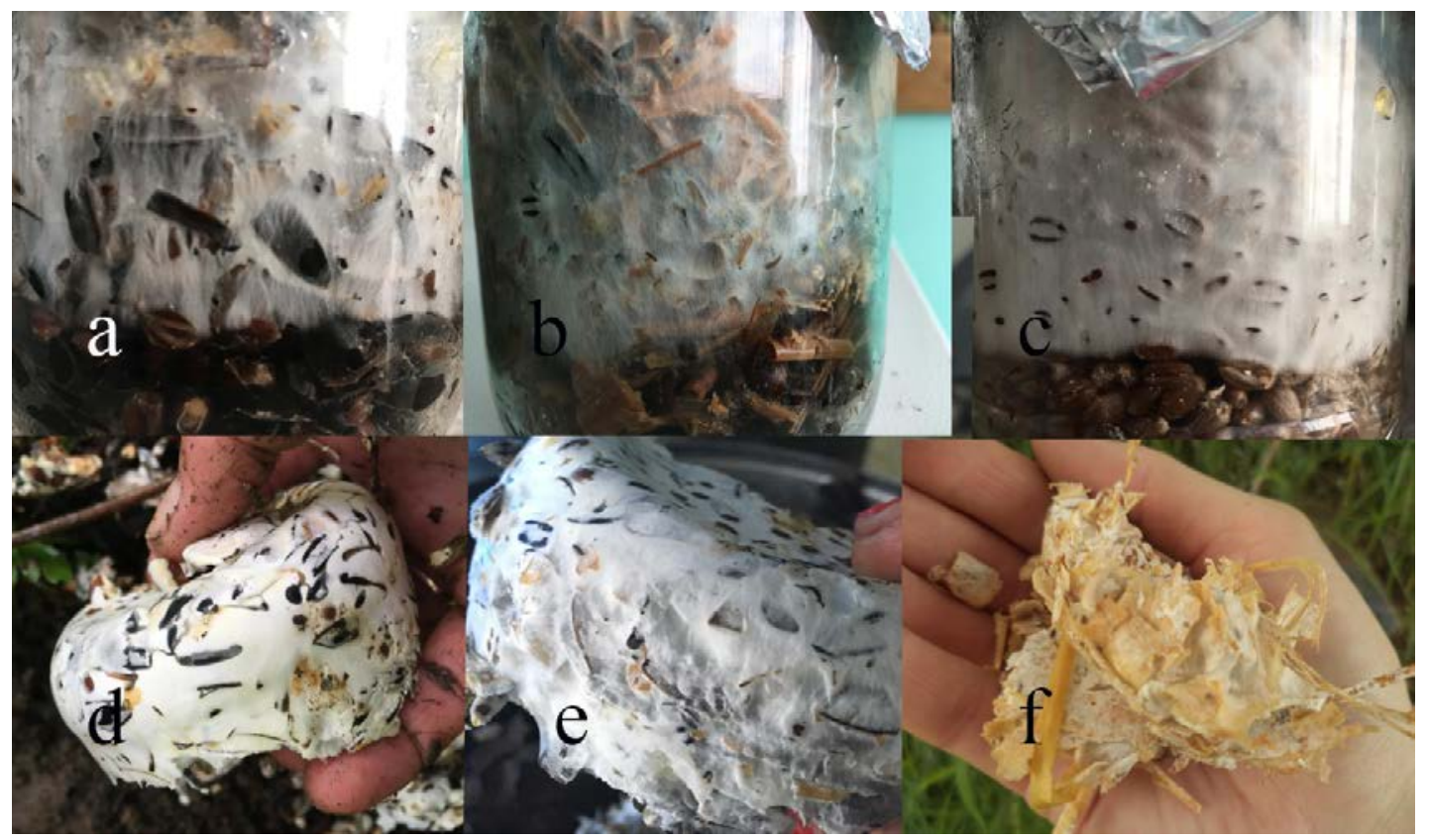

Fig. 6 - Polyporus umbellatus mycelium on the combined substrates. a, b, c Mycelium in the jars. $\mathrm{d}$, e Mycelium taken out from the jars. $\mathrm{f}$ Mycelium taken out from the flask.

\section{Morphology and growth rate of Polyporus umbellatus on plant leaf and WsOl substrates}

Polyporus umbellatus 2510 and 2511 are growing better on leaf substrate than on WsOl substrate. The level mycelial colonization of WsOl substrate was only $45-50 \%$ even after 30 days of cultivation (Figs 7, 8). Color of mycelial colonies on the leaf substrate was white or milky-white, covered with distinct spidery hyphae, while the center of the colony was more wooly (Fig. 9a, c). The mycelial colonies on the WsOl substrate was like a white cottage cheese (Fig. 9b, d).

\section{Primordia and fruiting bodies of Polyporus umbellatus formed on plant substrates}

Strain 2511 of $P$. umbellatus colonized the leaf substrate and WsOl substrate and its primordia and young fruiting bodies were developed on the day 18 and 38 accordingly (Fig. 10).

The fruiting bodies were formed on top of the bags on two substrate types (Fig. 10c, d). Spherical primordia with well-visible small numerous branches were formed at the perforation 
point of the bags (Fig. 10a, b). Young fruiting bodies had milky white color and became light gray while mature. The maximum size of the fully formed fruit bodies did not exceed $2.5 \times 3.5 \mathrm{~cm}$, weighing 60-70 g each, only 1-2 fruiting bodies per bag (at the perforation site) were formed. The fruiting bodies on top of the bags were composed of numerous caps with a barely noticeable stems. Caps had a common base, the size of fruit bodies didn't exceed 6-7 cm (basis) and 3-4 cm (high) (Fig. 10c). Within the same bag with leaf substrate we observed their simultaneous formation at both locations, i.e. at the perforation site and on the top of bag.

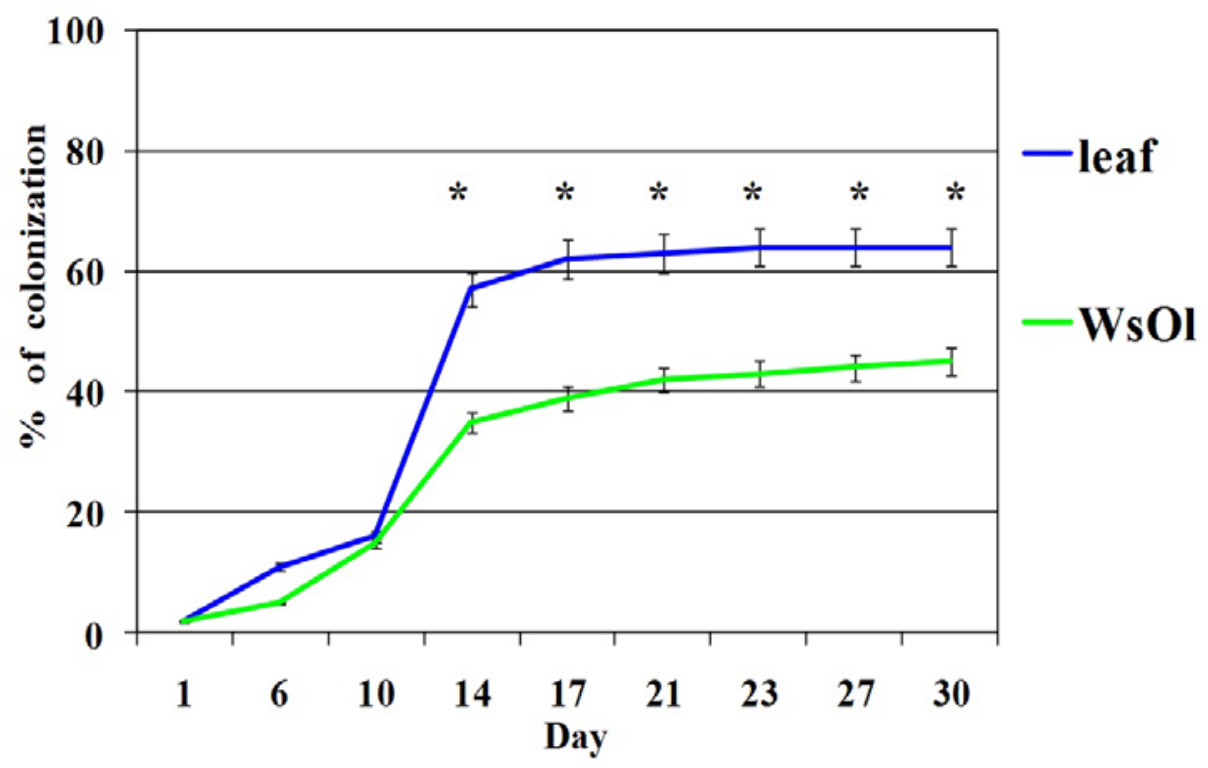

Fig. 7 - Colonization of plant substrates by the mycelium of Polyporus umbellatus 2510.

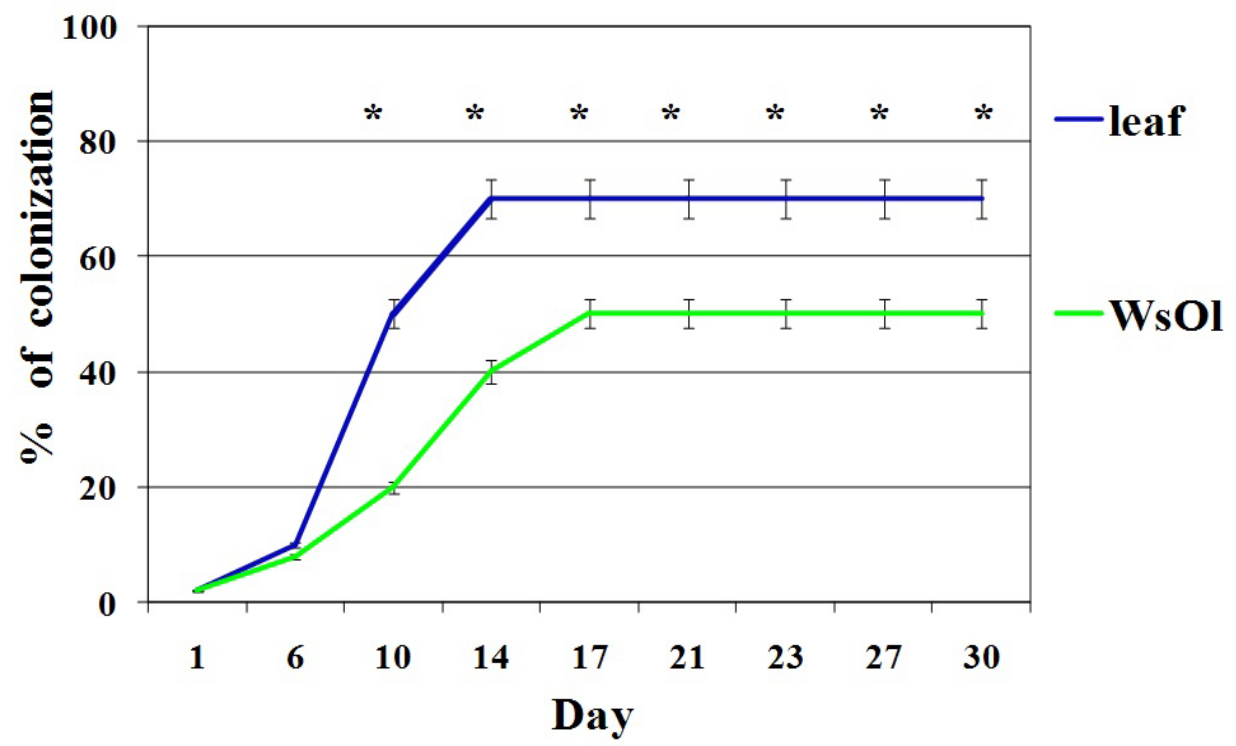

Fig. 8 - Growth rate of Polyporus umbellatus 2511 on plant substrates.

On the WsOl substrate the light gray spherical primordia of Polyporus umbellatus 2511 were formed only at the top of the package (Fig. 10d). Their lower part was lacking any patterns, with bushy branches on the top. The fruiting of P. umbellatus 2511 on WsOl substrate occurred only in $50 \%$ of cases, in such cases only one primordium was observed within one bag. Strain 2510 was not fruiting on any used substrate. 


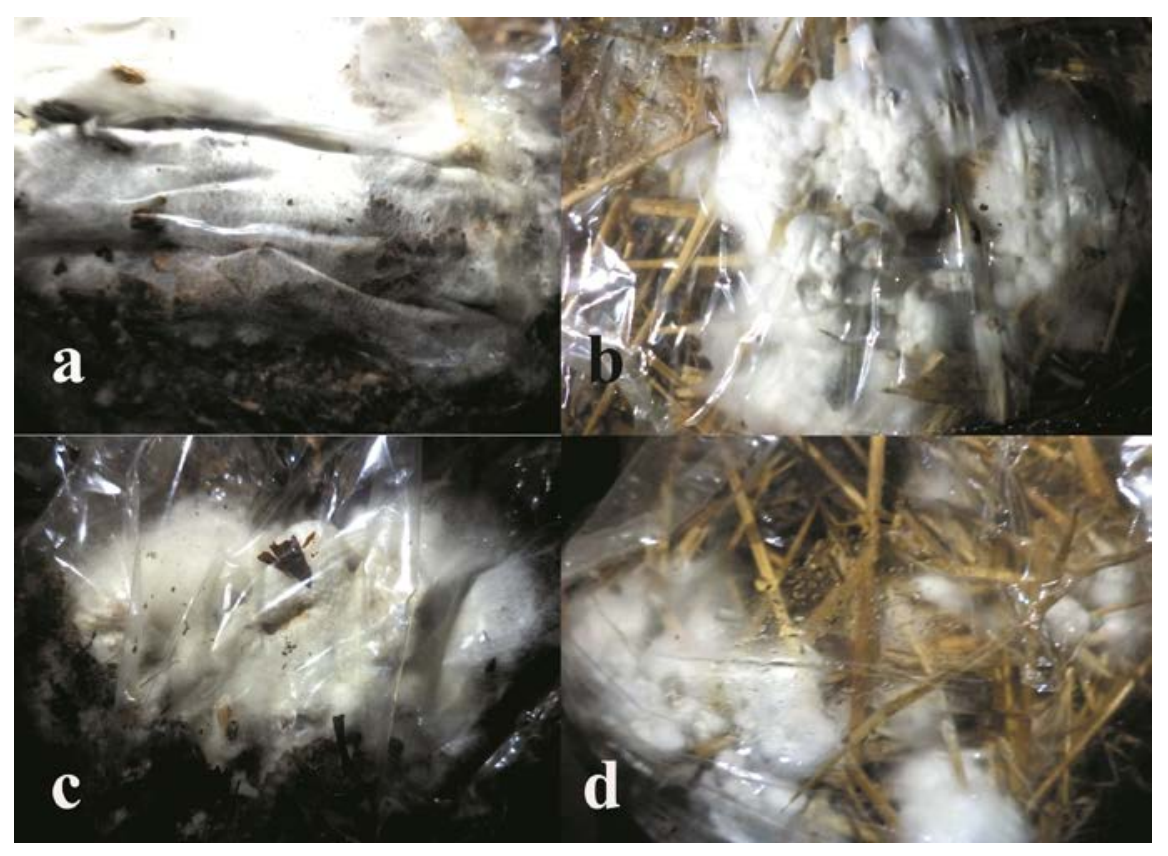

Fig. 9 - Polyporus umbellatus mycelium. a, b Strain 2511. c, d Strain 2510. a, c Leaf substrate. b, d WsOl substrate at day 23.

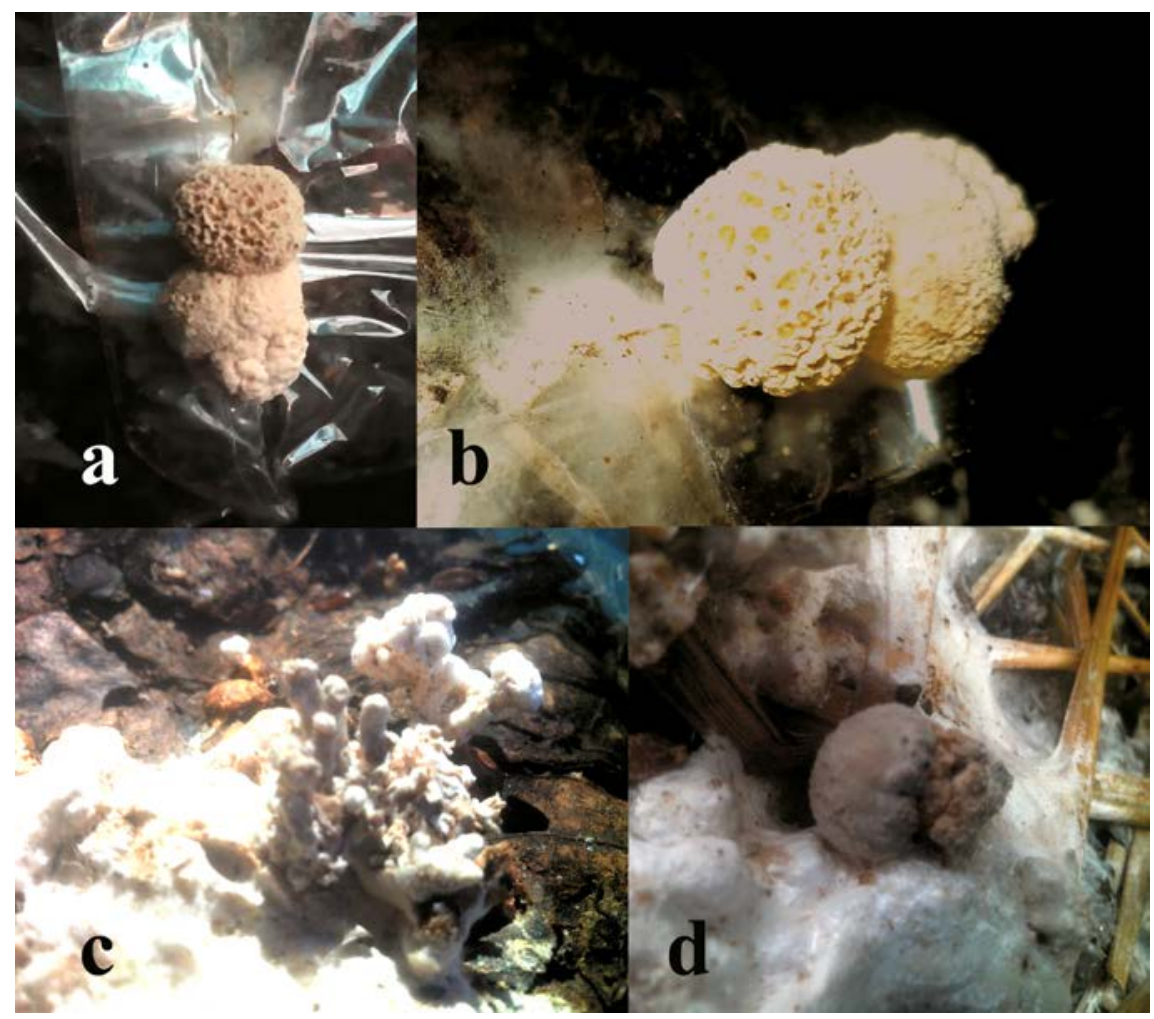

Fig. 10 - The primordia and fruiting bodies of Polyporus umbellatus strain 2511. a, b, c Mycelial colonization of the leaf substrate at day 18. $d$ Mycelial colonization of the WsOl substrate at day 38.

\section{Discussion}

The ecological distribution of the edible and medicinal mushroom P. umbellatus in Central Europe was studied. Main motivation of this investigation was to describe the potential for commercial cultivation of this species (Kunca \& Pavlik 2019). Xing et al. (2013) illustrated that exposure to low temperatures induced the $P$. umbellatus sclerotial morphogenesis during 
cultivation in a sawdust substrate. The low temperature treatment enhanced reactive oxygen species in mycelia, which may be important in triggering sclerotial differentiation in $P$. umbellatus. We demonstrated the $P$. umbellatus strain 2511 grew well at $22-26^{\circ} \mathrm{C}$ on the combinations of plant substrates. Kunca \& Pavlik (2019) reported that $P$. umbellatus predominantly grew in acidic soils (pH 4.5-4.9). Leaf and WsOl substrates have acidic $\mathrm{pH}$, this feature is important for $P$. umbellatus fruiting. However, $P$. umbellatus strain 2510 was not forming the fruiting bodies, which might be a sign of the culture degeneration. Also, the fruiting bodies of $P$. umbellatus strain 2511 had lighter weight than those found in natural habitats (2.5 to $3 \mathrm{~kg}$ ) (Petrichuk \& Pasailiuk 2015). Mycelium of both $P$. umbellatus strains obtained on WgBsWsPs could be stored for six months at $21^{\circ} \mathrm{C}$, without drops of exudate and yellowing of mycelium. Interestingly, the texture and degree of grinding of the substrate's components has a little influence on mycelial growth as it was observed for other fungi (Pasailiuk et al. 2018, 2019).

This work to find out the optimal weight ratio of the substrates and modifying them to continue. We do not exclude that modifying may change the time of overgrowth of substrates by mycelium of the fungus. We also see the prospect of future research in the application of the obtained knowledge for the remediation of the fungus in natural conditions by the re-situ method to preserve the specie in nature. Interesting, growing of $P$. umbellatus on the combination of plant substrates do not needs the Armillaria mellea mycelium like burying method or root inoculation method (Choi et al. 2003). Nevertheless, the obtained results are valuable because the sequence of affordable and relatively inexpensive plant substrates were found (fallen leaves are an almost inexhaustible resource), on which the fruiting of $P$. umbellatus 2511 can be achieved under laboratory conditions.

\section{Conclusion}

We obtained fructification of $P$. umbellatus on several plant substrates in axenic culture, without adding of A. mellea. However, one of two studied strains didn't fruit on any used substrate. Obtained fruitbodies were significantly smaller and not well developed comparing to the harvested in the natural habitat ones.

\section{Acknowledgements}

The author thanks Andrii Gryganskyi for editorial assistance with the manuscript.

\section{References}

Bandara AR, Rapior S, Bhat DJ, Kakumyan P et al. 2015 - Polyporus umbellatus, an ediblemedicinal cultivated mushroom with multiple developed health-care products as food, medicine and cosmetics: a review. Cryptogamie, Mycologie 36(1), 3-43.

Bisko NA, Lomberg ML, Mytropolska NY, Mykchaylova OB. 2016 - The IVC mushroom culture collection. Alter press, Kyiv.

Bisko NA, Sukhomlyn MM, Mykchaylova OB, Lomberg ML et al. 2018 - Ex situ conservation of rare and endangered species in mushroom culture collections of Ukraine. Ukrainian Botanical Journal 75(4), 338-347.

Bohlin A, Senn-Irlet B, Evans S, Kovalenko A et al. 2006 - European council for the conservation of fungi. ECCF, Newsletter.

Choi KD, Lee KT, Shim JO, Lee YS et al. 2003 - A new method for cultivation of sclerotium of Grifola umbellata. Mycobiology 31(2), 105-112.

Didukh YP. 2009 - Red data book of Ukraine. Vegetable Kingdom. Globalkonsaltyng, Kyiv.

Guo N, Bai Z, Jia W, Sun J et al. 2019 - Quantitative analysis of polysaccharide composition in Polyporus umbellatus by HPLC-ESI-TOF-MS. Molecules 24(14), 2526.

Guo S, Wang Q, Zhuang W, Zhang J, Xing X. 2002 - Discovery and application of the companion fungus related to sclerotial formation from hyphae of Grifola umbellata. Acta Botanica Sinica 44, 1151-1154. 
Huang HC, Liu YC. 2007 - Acceleration of mycelia growth by adding Grifola umbellata broth concentrate in solid-state and submerged cultures. Biochemical Engineering Journal 37, 139143.

Kotlaba F. 1984 - Geographical distribution and ecology of polypores (Polyporales s.l.) in Czechoslovakia, Praha.

Kunca V. 2011 - Ecology and incidence of Polyporus umbellatus in Slovakia. Czech Mycology 63(1), 39-53

Kunca V, Pavlik M. 2019 - Fruiting body production of, and suitable environmental ranges for, growing the umbrella polypore medicinal mushroom, Polyporus umbellatus (Agaricomycetes), in natural conditions in central Europe. International Journal Medicinal Mushrooms 21(2), 121-129.

Lee MW, Chang KC, Shin DB, Lee KR et al. 2013 - The culture conditions for mycelial growth and sclerotial formation of Polyporus umbellatus. Journal of Mushroom Science and Production 11(4), 194-200.

Liu Z, Liu Z. 2009 - Essentials of Chinese medicine, Springer, Verlag, London.

Lomberg ML, Solomko EF. 2012 - Growth of macromycetes cultures on agarized nutrient media and dense substrates. AlterPres, Kyiv.

Pasailiuk MV, Petrichuk YV, Tsvyd NV, Sukhomlyn MM. 2018 - The aspects of reproduction of Clathrus archeri (Berk.) Dring by the re-situ method in the National Nature Park Hutsulshchyna. Leśne Prace Badawcze 79(3), 287-293.

Pasailiuk M, Sukhomlyn M, Gryganskyi A. 2019 - Biological features of Sparassis laminosa (Sparassidaceae, Polyporales) and the main aspects of its reproduction in the territory of the Hutsulshchyna National Natural Park, Ukraine. Current Research in Environmental \& Applied Mycology 9(1), 194-207.

Petrichuk Y, Pasailiuk M. 2015 - New records of the fungi listed in the Red Data Book of Ukraine from the Pokutski Carpathians. Ukrainian Botanical Journal 72(4), 381-384.

Ryvarden L, Gilbertson RL. 1994 - European Polypores 2, Fungiflora, Oslo, Norway.

Ryvarden L, Melo I. 2014 - Poroid fungi of Europe. Synopsis Fungorum, Fungiflora.

Stalpers JA. 1978 - Identification of wood-inhabiting aphyllophorales in pure culture. Studies in Mycology 16, 1-248.

Stamets P. 2002 - Mycomedicinals: An informational treatise on mushrooms, MycoMedia, Olympia, Washington.

World Data Center for Microorganism. 2019 - Word Federation for Culture Collection. Culture Collection Information Worldwide. http://www.wfcc.info/ccinfo/index.php/collection/ by_id/1152/ (accessed 22 December 2019).

Wu JN. 2005 - An illustrated Chinese materia medica, Oxford University Press, New York.

Xing YM, Zhang LC, Liang HQ, Lv J et al. 2013 - Sclerotial formation of Polyporus umbellatus by low temperature treatment under artificial conditions. PLos One 8(2), e56190.

Yang L, Wang R, Liu J, Tong H et al. 2004 - The effect of Polyporus umbellatus polysaccharide on the immunosuppression property of culture supernatant of S180 cells. Chinese Journal of Cellular and Molecular Immunology 20, 234-237.

Ying J, Mao X, Ma Q, Zong Y. Wen H. 1987 - Icones of medicinal fungi from China, Science Press, Beijing, China.

Zeng X, Li CX, Huang Y, Zhang GW et al. 2011 - Effects of Polyporus umbellatus and Polyporus polysaccharide on the phagocytosis function and costimulatory molecules expression of peritoneal macrophages in rat bladder cancer. Chinese Journal of Immunology 27, 414-418.

Zhao JD, Zhang XQ. 1992 - The Polypores of China. Bibliotheca Mycologica 145, 521-524.

Zhao YY, Zhao YM, Lin RC, Sun WJ. 2009 - Qualitative and quantitative analysis of the diuretic component ergone in Polyporus umbellatus by HPLC with fluorescence detection and HPLCAPCI-MS/MS. Pharmazie 64, 366-370. 